\title{
Pancytopenia with Hemophagocytic Syndrome Associated with Histoplasmosis in Acquired Immunodeficiency Syndrome: Description of 2 Case Studies and Literature Review
}

\author{
Deeptarka Roy ${ }^{1}$, Pradipta Guha ${ }^{2 *}$, Dipanjan Bandyopadhyay ${ }^{3}$, Partha Sardar ${ }^{4}$ and Sanjoy Kumar Chatterjee ${ }^{5}$ \\ ${ }^{1}$ Deeptarka Roy, Medical College, Kolkata 88, College Street, Kolkata-700073, West Bengal, India \\ ${ }^{2}$ Pradipta Guha Calcutta National Medical College, Kolkata, 24, Gorachand Road, Kolkata - 700014, West Bengal, India \\ ${ }^{3}$ Dipanjan Bandyopadhyay Medical College, Kolkata 88, College Street, Kolkata-700073, West Bengal, India \\ ${ }^{4}$ Partha Sardar Lal Bahadur Shastri Hospital ,Khichripur, New Delhi-110091, India \\ ${ }^{5}$ Sanjoy Kumar Chatterjee Medical College, Kolkata 88, College Street, Kolkata-700073, West Bengal, India
}

\begin{abstract}
Two cases of haemophagocytic syndrome (HPS) due to histoplasmosis in patients with the acquired immunodeficiency syndrome (AIDS) are described in our case studies. Both the patients presented with pancytopenia and the bone marrow aspirate showed features suggestive of haemophagocytosis and the presence of intracellular inclusions were compatible with Histoplasma capsulatum. Haemophagocytic syndrome due to disseminated histoplasmosis should be included in the differential diagnosis of pancytopenia in patients with AIDS, though it is a rare clinical entity.
\end{abstract}

Keywords: Pancytopenia; Hemophagocytic syndrome; Histoplasmosis; HIV infection

\section{Introduction}

Histoplasmosis, caused by the fungus Histoplasma capsulatum, is also known as Darling's disease. Two varieties of this fungus, $\mathrm{H}$. capsulatum var. capsulatum and $\mathrm{H}$. capsulatum var. duboisii, are pathogenic to humans [1] Disseminated histoplasmosis is associated with Acquired Immunodeficiency Syndrome (AIDS), involves different organ systems and may be fatal if untreated [2,3]. Bone marrow histoplasmosis is a rare manifestation of disseminated Histoplasma infection presenting as pancytopenia. We report two such cases to document this rare but definitive cause of pancytopenia in the setting of advanced immunodeficiency.

\section{Case 1}

A 35 year old unmarried truck driver presented to us with generalized weakness and fever for the past 6 months, along with skin rashes for the past 2 weeks. The weakness was insidious in onset, gradually progressive, and accompanied with pallor, exertional shortness of breath and palpitation. This was associated with low grade, intermittent fever for the same duration without any chills or rigors. The skin rashes were non pruritic. He was diagnosed to harbor HIV infection 11 years earlier, but had been under regular medical care for the last 4 months.Disseminated tuberculosis (TB) was diagnosed 4 months back and he has been on CAT 1 Anti Tubercular Drugs (ATD) since then.

General examination revealed drowsy cachectic patient, with profound pallor, multiple non-tender cervicallymph nodes, hypotension and fever of $99.6^{\circ} \mathrm{F}$. Dermatological survey revealed multiple petechiae and ecchymotic patches. Firm, non-tender hepatosplenomegaly was evident on abdominal survey. Sternal and joint tenderness were absent. We made a provisional diagnosis of hypoplastic anemia, possibly due to marrow tuberculosis. A lympho-proliferative disorder remained a close differential diagnosis

Blood counts revealed pancytopenia (Hb 4.4 gm\%, TC 1400 cells/cumm, Plt 14,000 cells/cumm) with a raised ESR of $140 \mathrm{~mm} / \mathrm{hr}$. The anemia was normocytic and normochromic in nature. Altered albumin-globulin ratio and raised ALP $(1610 \mathrm{IU} / \mathrm{l}$, normal $<430 \mathrm{IU} / \mathrm{l})$ were the highlights of the Liver Function Test. Other biochemical tests were normal. Ultrasound confirmed hepatosplenomegaly along with peripancreatic lymphadenopathy. Adrenal glands were normal Mantoux was negative at $4 \mathrm{~mm}$. The CD4 counts at diagnosis of HIV and presentation were 250 cells $/ \mu \mathrm{L}$ (1998) and 29 cells/ $\mu \mathrm{L}$ (2009) respectively. Viral markers for Hepatitis B and C were negative, while the HIV-1 Viral Load was 257693 copies / ml. FNAC from a cervical node demonstrated epitheloid granuloma without caseation and no Acid Fast Bacilli (AFB). Biopsy was abandoned in view of a raised Bleeding Time. Bone marrow histopathology showed a hypocellular marrow with intact $\mathrm{M}: \mathrm{E}$ ratio. Bone marrow biopsy aspirate shows phagocytic histiocyte with ingested Histoplasma yeast bodies and selfhematopoietic cells-findings that are suggestive of hemophagocytic syndrome .In addition groups of budding yeast cells were also seen within reticulin cells, providing us with a confirmatory diagnosis of bone marrow Histoplasmosis (Figures 1a and $1 \mathrm{~b}$ ).

The patient was started on I.V. Amphotericin B therapy with daily monitoring of serum creatinine and potassium levels. Transfusion support was maintained as was ATD and Co-trimoxazole prophylaxis. However the patient succumbed to his illness on $12^{\text {th }}$ day of antifungal therapy.

\section{Case 2}

This 41 year old male was a married trucker presenting with generalized weakness for 4 months followed by fever and diarrhoea

*Corresponding authors: Pradipta Guha, Senior Resident, Department of cardiology, Calcutta National Medical College, Kolkata Address, Nandan Nagar, Belgharia, Kolkata 700083, West Bengal, India, Tel: 091-33-25412415; E-mail: dr.pradipta@yahoo.co.in

Received November 20, 2010; Accepted December 31, 2011; Published January 10, 2011

Citation: Roy D, Guha P, Bandyopadhyay D, Sardar P, Chatterjee SK (2011) Pancytopenia with Hemophagocytic Syndrome Associated with Histoplasmosis in Acquired Immunodeficiency Syndrome: Description of 2 Case Studies and Literature Review. J AIDS Clinic Res 2:115. doi:10.4172/2155-6148.1000115

Copyright: (c) 2011 Roy D, et al. This is an open-access article distributed unde the terms of the Creative Commons Attribution License, which permits unrestricted use, distribution, and reproduction in any medium, provided the original author and source are credited. 
Citation: Roy D, Guha P, Bandyopadhyay D, Sardar P, Chatterjee SK (2011) Pancytopenia with Hemophagocytic Syndrome Associated with Histoplasmosis in Acquired Immunodeficiency Syndrome: Description of 2 Case Studies and Literature Review. J AIDS Clinic Res 2:115. doi:10.4172/2155-6148.1000115

Page 2 of 3
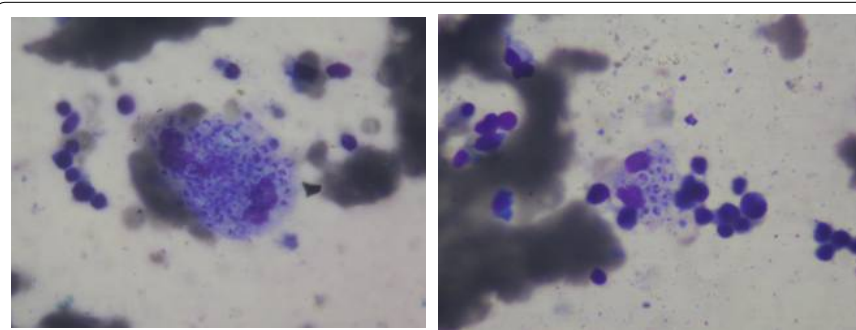

Figure: 1a \& b Bone marrow aspirate showing a phagocytic histiocyte with ingested Histoplasma yeast bodies intracellular yeast forms consistent with Histoplasma capsulatum and self-hematopoietic cells-findings that are suggestive of hemophagocytic syndrome (hematoxylin-eosin, original magnification $\times 400$ ).
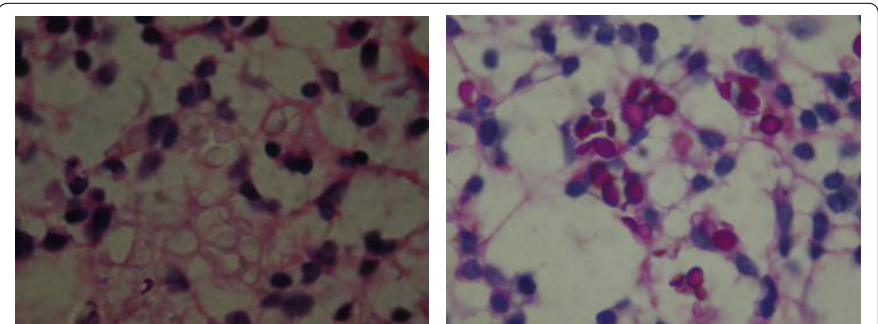

Figure: 2a \& b Bone marrow biopsy revealing extensive involvement by Histoplasma capsulatum (PAS stain, original magnification $\times 400$ ).

for last 3 months. Fever was low grade and not associated with chills, rigor or sweating. The diarrhoea was persistent over 3 months with passage of 5-6 watery stools per day. Past medical history included Pulmonary TB diagnosed 2 years back for which ATD was initiated but discontinued at 2 months of therapy. Personal history included commercial sexual exposures, 15 pack years of smoking and occasional alcohol intake.General survey documented wasting, severe pallor, tachycardia, tachypnoea, high temperature and oral candidiasis. Hemogram revealed pancytopenia with high ESR. The peripheral blood smear demonstrated normochromic normocytic RBCs, with the corrected reticulocyte index of 1.5. Blood biochemistry was normal except for raised ALP and LDH levels. Mantoux was $12 \mathrm{~mm}$ at 72 hours. Bone marrow HPE showed hypocellular marrow with intact M:E ratio. HIV serology was positive and the CD4 count was 36cells/ $\mu \mathrm{L}$. Serology for HBV, HCV and Syphilis were negative. Stool for OPC was unyielding. Blood culture grew colonies of S. pneumoniae .A provisional diagnosis of pneumococcal septicemia with sepsis induced bone marrow suppression was arrived at with disseminated TB being high on our minds.

The patient was started on I.V. antibiotics with oral Fluconazole. Transfusion support and Co-trimoxazole prophylaxis were initiated. The patient continued to be pale, febrile and toxic. Disseminated TB was considered and empiric ATD was instituted. The hematologist suggested a bone-marrow biopsy. The marrow biopsy specimen demonstrated budding yeast cells within macrophages, suggestive of Histoplasma capsulatum (Figures 2a \& 2b).

I.V. Amphotericin B was initiated with proper metabolic monitoring. The patient improved clinically, fever subsided and the diarrhoea was controlled. He has been on follow-up at our HIV clinic and is stable on Highly Active Anti Retroviral Therapy (HAART).

\section{Discussion}

Infection with Histoplasma is asymptomatic in $95 \%$ of cases. However the clinical picture can be strikingly different in AIDS, with disseminated histoplasmosis being a high possibility [4]. Patients with CD 4 counts of $<150$ cells $/ \mu$ are at most risk [2,3].Disseminated histoplasmosis can be manifested as hepatosplenomegaly, lymphadenopathy, gastrointestinal involvement, skin lesions, renal failure, acute respiratory distress and disseminated intravascular coagulation. Unique manifestations include adrenal gland involvement, ocular involvement, reactive haemophagocytic syndrome and bone marrow histoplasmosis [2].

Disseminated TB is a close differential for histoplasmosis. A report from Guatemala showed that of the 8 patients who had disseminated histoplasmosis with AIDS, all were initially admitted with diagnosis of tuberculosis with AIDS [5]. Even in both the cases reported above, ATD was provisionally instituted prior to the diagnosis of histoplasmosis.

Bone marrow involvement in disseminated histoplasmosis may present as Pyrexia of Unknown Origin or unexplained pallor with or without other manifestations. Tissue should be stained with methenamine silver or periodic acid-Schiff stains to best visualize $\mathrm{H}$. capsulatum. Yeasts are typically found within macrophages but can also be seen free in tissues [6]. Kurtin et al. [7] studied the bone marrow in 13 patients with AIDS. Multiple granulomas, diffuse macrophage infiltrates and lymphohistiocytic aggregates were the common morphological picture though two patients did not reveal any morphological evidence of infection. Morphologic examination of the bone marrow with special fungal stains combined with cultures is useful in diagnosing disseminated histoplasmosis [7].

Classically, histoplasmosis has been treated with long courses of amphotericin B. The current practice is to use lipid formulations of amphotericin B in most patients because of their reduced toxicity. For almost all patients, as their condition improves their therapy is switched to oral itraconazole. Studies have shown a very low risk of relapse when $\mathrm{CD} 4$ counts have remained at $>200$ cells/ $\mu$ l.The current practice is to discontinue antifungal therapy for patients achieving this level of CD4 reconstitution with anti-retroviral therapy [8]. With the use of effective antiretroviral therapy, rates of histoplasmosis have fallen in the AIDS population, and prophylaxis is no longer recommended.

We want to highlight this case studies since, Haemophagocytic syndrome due to disseminated histoplasmosis should be included in the differential diagnosis of pancytopenia in patients with AIDS, though it is a rare clinical entity. So we should remain vigilant regarding this fact during evaluation of patient with HIV. Proper evaluation and early initiation of therapy may alter the life expectancy of these patients.

\section{Acknowledgement}

Dr.Deeptarka Roy and Dr.Pradipta Guha contributed equally in this work.

Conflict of Interest: None

Funding Source: None

Ethical Approval: Taken

\section{References}

1. Kauffman CA, Histoplasmosis.. In Dismukes WE, Pappas PG, and Sobel JD (2003) Clinical mycology. Oxford University Press, New York, NY.

2. Gutierrez ME., Canton A., Sosa N, Puga E, Talavera L (2005) Disseminated histoplasmosis in patients with AIDS in Panama: a review of 104 cases. Cli Infect Dis 40: 1199-1202.

3. McKinsey DS., Spiegel RA., Hutwanger L., Stanford J, Driks MR et al. (1997) Prospective study of histoplasmosis in patients infected with human immunodeficiency virus: incidence, risk factors, and pathophysiology. Clin Infect Dis 24:1195-1203.

4. Wheat LJ, Connolly-Stringfield PA., Baker RL, Curfman MF, Eads ME et al (1990) Disseminated histoplasmosis in the acquired immune deficiency 
Citation: Roy D, Guha P, Bandyopadhyay D, Sardar P, Chatterjee SK (2011) Pancytopenia with Hemophagocytic Syndrome Associated with Histoplasmosis in Acquired Immunodeficiency Syndrome: Description of 2 Case Studies and Literature Review. J AIDS Clinic Res 2:115. doi:10.4172/2155-6148.1000115

syndrome: Clinical findings, diagnosis and treatment and review of literature. Medicine (Baltimore) 69: 361-374

5. Segura L, Rojas M, Pelaez N, Shor-Posner G, RosaRe D et al. (1997) Disseminated histoplasmosis and human immunodeficiency virus type 1 infection: risk factors in Guatemala. Clin Infect Dis 25: 343-344.

6. Cooperberg AA, and Schwartz J (1964) The diagnosis of disseminated histoplasmosis from marrow aspiration. Ann Intern Med 61: 289-295.
7. Kurtin PJ, McKinsey DS, Gupta MR, Driks M, et al. (1990) Histoplasmosis in patients with acquired immunodeficiency syndrome. Hematologic and bone marrow manifestations. Am J Clin Pathol 93: 367-372.

8. Goldman M, Zackin R, Fichtenbaum CJ, Skiest DJ, Koletar SL, et al (2004) Safety of discontinuation of maintenance therapy for disseminated histoplasmosis after immunologic response to antiretroviral therapy. Clin Infect Dis 38:1485-1489. 